Journal of Patient-Centered

Volume 4

Issue 4 -- Health Disparities and Inequities: Part

Article 21

I

$11-6-2017$

\title{
Improving Obstetrics in Family Medicine Residency Clinics: A Quality Improvement Study
}

Garima Chawla

Jessica J.F. Kram

Bonnie Bobot

Dennis J. Baumgardner

Follow this and additional works at: https://aah.org/jpcrr

Part of the Family Medicine Commons, Medical Education Commons, Obstetrics and Gynecology Commons, and the Women's Health Commons

\section{Recommended Citation}

Chawla G, Kram JJ, Bobot B, Baumgardner DJ. Improving obstetrics in family medicine residency clinics: a quality improvement study. J Patient Cent Res Rev. 2017;4:260-1.

Published quarterly by Midwest-based health system Advocate Aurora Health and indexed in PubMed Central, the Journal of Patient-Centered Research and Reviews (JPCRR) is an open access, peer-reviewed medical journal focused on disseminating scholarly works devoted to improving patient-centered care practices, health outcomes, and the patient experience. 
for patient characteristics, device specifications and indicators of post-THA complication (serum $\mathrm{Cr}$ ion, $\mathrm{Co}$ ion, C-reactive protein, erythrocyte sedimentation rate).

Results: In total, 162 hips and 152 unique patients underwent THA during September 2009-May 2012, with 78 hips subsequently revised during 2012-2015. Patients were of median age 62 years (range: $32-$ $90)$, nearly all non-Hispanic white (89\%) and mostly female (58\%). Several variables were significantly associated $(\mathrm{P}<0.05)$ with ALTR grade, including occurrence of complication symptoms (eg, pain), patient age, and Co ion concentrations. Revision surgery was the most strongly associated variable with ALTR, with 5 times greater odds of abnormal grade when not undergoing revision (odds ratio: 5.68, 95\% confidence interval: 2.69-11.9).

Conclusion: Within AHC, patients who underwent THA with the Stryker Rejuvenate hip implant often experienced the complications of ALTR, but revision surgery reduced the ALTR grade.

\section{Family Practice Resident Expectations by Year From Faculty and Resident Perspectives: A Quality Improvement Initiative}

Alyssa Krueger, Devin Lee, Jessica J.F. Kram, Will Lehmann, Dennis J. Baumgardner

Department of Family Medicine, Aurora University of Wisconsin Medical Group; Center for Urban Population Health

Background: The transition from student to physician requires substantial commitment and work from residents as well as guidance from program faculty. The Accreditation Council for Graduate Medical Education (ACGME) has standardized certain academic requirements for U.S. residency programs; however, faculty expectations of residents according to year in the program are less formal and more a hidden curriculum. Setting expectations for residents to consult could better help residents navigate their graduate medical education experience and achieve the level of excellence expected by ACGME. Purpose: Our quality improvement study aimed to: 1) determine what the expectations of family practice residents were based on feedback from faculty members and current residents; and 2) share these expectations with residents.

Methods: A preintervention survey was emailed to family medicine program faculty and residents regarding resident expectations according to year in the program. Based on the results of the preintervention survey, expectations were outlined in a handout according to year in the program and were presented to current residents during scheduled didactic time. Residents who responded to the preintervention survey were then asked to respond to the postintervention survey. Fisher exact tests were used to compare preand postintervention survey responses.

Results: Overall, 64\% (14 of 22) of faculty and 64\% (18 of 28) of residents responded to the preintervention survey. While $79 \%$ of faculty expressed that they had specific expectations for residents, $77 \%$ felt that residents did not know these expectations. Additionally, while residents (94\%) believed faculty had expectations of them, only $33 \%$ knew what the expectations were. Following intervention, 15 of 18 residents responded, with $79 \%$ now reporting they knew what the expectations were $(\mathrm{P}=0.02)$. The handout was found useful by all those queried, and $85 \%$ felt it clarified expectations.

Conclusion: At baseline, residents and faculty knew there were expectations for residents as they progress through the program, but those expectations were not explicit. Despite the lack of vertical communication, the expectations from both groups were surprisingly similar. A handout delivered electronically and at didactic sessions was deemed useful and clarified expectations.

The Lifestyle Initiative: An Innovative Coaching-Based Quality Improvement Study to Improve the Health of Aurora Health Care Caregivers and Family Members

\author{
Tiffany Mullen, Jessica J.F. Kram, Dennis J. Baumgardner
}

Department of Integrative Medicine, Aurora Sinai Medical Center; Department of Family Medicine, Aurora University of Wisconsin Medical Group; Center for Urban Population Health

Background: Self-management support has been shown to improve clinical outcomes. Health coaching, one form of self-management support, empowers patients within the health care system by providing information and through collaboratively developed care plans.

Purpose: Assess the impact of The Lifestyle Initiative, a coachingbased health program utilized by Aurora Health Care caregivers or family members.

Methods: The Lifestyle Initiative is a coaching-based approach for Aurora caregivers or family members enrolled in Aurora's health insurance network. Individuals were recruited from the care management database, and all participants had an Aurora primary care provider. Participation was limited to those $\geq 18$ years of age who had a diagnosis of type 2 diabetes or hypertension (or both), had glycated hemoglobin $(\mathrm{A} 1 \mathrm{c}) \leq 8.0$, and were not on insulin. The Lifestyle Initiative was rolled out in three phases. Phase I: health coaching sessions through a standard web- and app-based platform (Noom Health), and access to a stress-management program (HeartMath). Phase II: health coaching sessions through a standard web- and app-based platform co-created by Aurora's Department of Integrative Medicine and Noom Health, and access to HeartMath; Phase III: health coaching sessions through a standard web- and app-based platform (Noom Health), and a customized web- and app-based platform. Those enrolled in each phase acted as their own controls. Paired t-tests were used to compare pre- and postintervention results of each phase.

Results: The majority of Phase I participants ( $\mathrm{n}=23$; mean age 54.4 years) were female $(91.3 \%)$ and white $(52.2 \%)$. Preintervention A1c and blood pressure were not statistically different postintervention. However, pre- vs postintervention weights were statistically different (228.2 vs $218.5 \mathrm{lb} ; \mathrm{P}<0.01$ ), as well as pre- vs postintervention body mass index ( 37.3 vs $\left.35.7 \mathrm{~kg} / \mathrm{m}^{2} ; \mathrm{P}<0.01\right)$. The majority of Phase II participants $(\mathrm{n}=63$; mean age 54.8 years) also were female $(81.0 \%)$ and white $(88.9 \%)$. Pre- and postintervention blood pressures were not statistically different. However, pre- vs postintervention A1c (7.2 vs 6.6; $\mathrm{P}<0.02$ ), weights ( 229.6 vs $225.7 \mathrm{lb} ; \mathrm{P}<0.05$ ), and body mass index ( 37.1 vs $36.5 \mathrm{~kg} / \mathrm{m}^{2} ; \mathrm{P}<0.05$ ) were statistically improved. Phase III data collection is underway.

Conclusion: The Lifestyle Initiative health coaching program significantly improves certain health metrics when applied to health system employees and family members with diabetes or hypertension. Further study is needed to explore sustainability and the effects of more robust programs.

Improving Obstetrics in Family Medicine Residency Clinics: A Quality Improvement Study

Garima Chawla, Jessica J.F. Kram, Bonnie Bobot, Dennis J. Baumgardner

Department of Family Medicine, Aurora University of Wisconsin Medical Group; Center for Urban Population Health 
Background: Prenatal care/deliveries within our family medicine clinics have declined, perhaps because patients are unaware that our clinics provide these services. With lower volumes, clinicians may feel less comfortable with current skills/knowledge of obstetric (OB) care. Purpose: Increase family medicine clinic OB numbers, patient awareness, and clinician comfort/knowledge in OB.

Methods: English-facile patients (18-50 years), residents and faculty at Aurora family medicine residency clinics were included. Patients were provided preintervention surveys upon check-in. Residents/ faculty were surveyed via Survey Monkey. Changes made based on initial survey results were: 1) increasing systemwide awareness that our caregivers provide $\mathrm{OB}$ care, through fliers at emergency departments/urgent cares or posters in clinic waiting rooms; 2) keeping at least one same-day visit for $\mathrm{OB}$ patients; 3 ) distributing standard $\mathrm{OB}$ note templates to residents/faculty; and 4) placing patient educational handouts at each clinic. Patients, residents and faculty were reassessed at 9 months postintervention. Surveys were analyzed with Fisher's exact tests.

Results: Respondents to the preintervention survey included 83 patients, 26 residents and 19 faculty; 61 patients, 23 residents and 21 faculty responded to the postintervention survey. On both preand post-surveys, patients knew that their providers delivered babies ( $59 \%$ vs $57 \%$, respectively; $\mathrm{P}=0.86$ ). However, only $22 \%$ and $33 \%$ of patients, respectively, had a doctor at our clinics deliver their baby or partner's baby $(\mathrm{P}=0.25)$. Even so, $95 \%$ and $100 \%$ of patients, respectively, would recommend their friends or family to our family practice clinics if they became pregnant $(\mathrm{P}=0.14)$. On the pre-survey, $38 \%$ of residents felt clinic OB numbers were adequate versus $70 \%$ following intervention $(\mathrm{P}<0.05)$. On both pre- and postsurveys, residents planned on incorporating obstetric or prenatal care into their future practice ( $42 \%$ vs $52 \%$, respectively; $\mathrm{P}=0.57$ ). On both pre- and post-surveys, faculty felt comfortable with $\mathrm{OB}$ skills and knowledge ( $53 \%$ vs $62 \%$, respectively; $\mathrm{P}=0.75$ ). Lifestyle was the most common reason faculty gave for why they stopped doing deliveries (37\% vs 33\%, respectively).

Conclusion: Implementation of changes to our OB workflow resulted in non-statistically significant improvements in viewpoints toward OB. Resident feelings of $\mathrm{OB}$ number adequacy significantly improved following intervention. Further study in multiple clinics could confirm the effectiveness and reasons for success of our interventions.

\section{Brain Imaging in Older Patients With Delirium}

\section{Laila M. Hasan, Ariba Khan, Maharaj Singh, Michael L. Malone}

\section{Department of Geriatrics, Aurora University of Wisconsin Medical Group; Aurora Research Institute}

Background: Delirium is a common, serious and costly condition in older patients admitted to the hospital. This study describes the prevalence and results of brain imaging among a cohort of older hospitalized patients with and without delirium.

Purpose: Investigate the frequency and results of brain imaging in older patients with delirium as compared to those without delirium. Methods: This was a cross-sectional study. Data were collected on hospitalized patients age 65 years or older who were admitted to 3 hospitals in Milwaukee, Wisconsin, during a 1-month period in the fall of 2013. Subjects were tested for delirium via the "Confusion Assessment Method" by researchers for another study. The collected data included demographics, presence of delirium, computed tomography (CT) and magnetic resonance imaging (MRI), and results of the imaging procedures. The imaging studies were done as a part of their medical care. The authors reviewed the radiologist's final readings of the imaging studies. For all categorical variables, chi-squared/Fisher's test was used with alpha of 0.05 .

Results: A total of 92 patients were included in the study. Prevalence of delirium was $17.4 \%$. Mean age was 77 years. Overall, $24 \%$ had a CT and $9 \%$ an MRI, with the most common abnormal finding being chronic microvascular changes (13\%). CT scan was performed in $44 \%$ of patients with delirium and $20 \%$ of patients without delirium $(\mathrm{P}=0.04)$. MRI was performed in zero patients with delirium and $11 \%$ without delirium $(\mathrm{P}=0.34)$. When patients with delirium were compared with patients without delirium, respectively: normal imaging was described in 1 vs 2 patients $(\mathrm{P}=0.70)$; cerebral atrophy in 3 vs $6(\mathrm{P}=0.99)$; chronic microvascular changes in 2 vs 10 $(\mathrm{P}=0.17)$; and acute hematoma (subdural or intraparenchymal) in 3 (43\%) vs $0(\mathrm{P}=0.02)$.

Conclusion: In this limited study, patients with delirium were noted to be more likely to have had a CT scan. Older patients with delirium had a variety of findings on brain imaging, some of which were more clinically relevant. No specific imaging changes were diagnostic for delirium.

\section{Quality Improvement Study for Postpartum Hypertension Readmissions}

Molly K. Lepic, Sara M. Stanenas, Carla J. Kelly, Deborah Simpson, Jeffrey A. Stearns

Department of Obstetrics and Gynecology, Aurora Sinai Medical Center, Aurora University of Wisconsin Medical Group; Departments of Family Medicine and Academic Affairs, Aurora Health Care

Background: Hospital readmission rates are a focus of the Centers for Medicare and Medicaid Services. This was identified as a system opportunity to improve health care quality and patient education in order to reduce preventable readmissions. In 2009, $27 \%$ of obstetric readmissions were due to hypertensive disease, and preventable readmissions regarding hypertension are flagged as an area for quality improvement in our health care system. There is limited evidence on specific management of postpartum hypertension.

Purpose: Identify risk factors in our community and reduce postpartum readmissions for hypertension within our hospital.

Methods: We performed a retrospective chart review from November 2014 to November 2015. We collected demographic data, comorbidities and information regarding hospitalization and readmission. In this, we identified 28 readmissions for postpartum hypertension, representing $57 \%$ of obstetric readmissions and noted that discharge instructions and blood pressure monitoring postpartum were two areas for improvement. Only 18\% had printed instructions regarding postpartum hypertension. Via multidisciplinary education sessions, we aimed to increased surveillance for postpartum vitals for at-risk patients and provide appropriate verbal and written precautions for signs and symptoms of de novo or worsening hypertensive disease. We also improved access to care by scheduling blood pressure checks within 72 hours of discharge and utilization of visiting nursing services for blood pressure checks. The same measures were then recollected for readmissions from June 2016 to December 2016.

Results: After intervention, $61 \%$ of readmissions were related to hypertension, with 31 readmissions. Overall, there was a significant improvement in written discharge instructions regarding postpartum hypertension, with $94 \%$ receiving written instructions. At discharge, $33 \%$ had blood pressure checks and 13\% had visiting nursing services arranged. 